\title{
Development of a Compact Photoacoustic Tomography Imaging System with Dual Single-Element Transducers for Image Enhancement"
}

\author{
Yong-jian ZHAO ${ }^{1,2 \dagger}$, Xiao-long ZHU ${ }^{1 \dagger}$, Pei-yu LUO ${ }^{1}$, Ang LI $^{2}$, Wei XIAO ${ }^{1}$, Xiao XIAO ${ }^{1}$, Li LIU ${ }^{2 \#}$, Max Q.-H. MENG ${ }^{1,2,3 \#}$ \\ ${ }^{1}$ Department of Electronic and Electrical Engineering, the Southern University of Science and Technology, Shenzhen 518055, \\ China \\ ${ }^{2}$ Department of Electronic Engineering, The Chinese University of Hong Kong, Hong Kong 999077, China \\ ${ }^{3}$ Shenzhen Research Institute of The Chinese University of Hong Kong in Shenzhen, Shenzhen 518172, China
}

(C) The Author(s) 2021

\begin{abstract}
[Abstract] Objective: This paper proposes a new photoacoustic computed tomography (PACT) imaging system employing dual ultrasonic transducers with different frequencies. When imaging complex biological tissues, photoacoustic (PA) signals with multiple frequencies are produced simultaneously; however, due to the limited bandwidth of a single-frequency transducer, the received PA signals with specific frequencies may be missing, leading to a low imaging quality. Methods: In contrast to our previous work, the proposed system has a compact volume as well as specific selection of the detection center frequency of the transducer, which can provide a comprehensive range for the detection of PA signals. In this study, a series of numerical simulation and phantom experiments were performed to validate the efficacy of the developed PACT system. Results: The images generated by our system combined the advantages of both high resolution and ideal brightness/contrast. Conclusion: The interchangeability of transducers with different frequencies provides potential for clinical deployment under the circumstance where a single frequency transducer cannot perform well.

Key words: photoacoustic tomography; dual transducers; image enhancement; signal responding range
\end{abstract}

The emerging biomedical imaging modality of photoacoustic imaging (PAI) has made remarkable developments in the past decades, and it holds strong potential in making a significant impact in the field of medicine ${ }^{[1,2]}$. It is a hybrid imaging method that combines the advantages of both optical imaging and ultrasound imaging ${ }^{[3,4]}$. When a biological tissue is exposed to a pulsed laser beam, it absorbs the energy and experiences a temperature increment. Afterwards, the tissue expands in volume and emits ultrasonic waves, which can be received by ultrasonic transducers as photoacoustic (PA) signals ${ }^{[5,6]}$. During this process, neither radiation nor poisonous agents are involved, and it is cost-effective to generate PA images ${ }^{[7,8]}$. In general, PAI is typically classified into several categories

Yong-jian ZHAO, E-mail: Yongjian.zhao1995@link.cuhk. edu.hk; Xiao-long ZHU, E-mail: zhuxl@sustech.edu.cn

†Yong-jian ZHAO and Xiao-long ZHU contributed equally to this article.

\#Corresponding authors, Li LIU, E-mail: liliu@cuhk.edu.hk; Max Q.-H. MENG, E-mail: max.meng@ieee.org

${ }^{*}$ This research was supported by National Key R\&D program of China (No. 2019YFB1312400), Hong Kong Health and Medical Research Fund (HMRF) (No. 06171066), and CUHK-Direct (No. 134997202). according to the various system configurations ${ }^{[9,10]}$; the one most suitable for large-scale organ imaging is known as photoacoustic computed tomography (PACT) due to its large imaging depth ${ }^{[11,12]}$. Specifically, it applies unfocused laser beams. When the laser beam is expanded to form a large light spot, the whole imaging object is well illuminated. The transducers are usually distributed on multiple positions surrounding the object in order to receive PA signals ${ }^{[6]}$. One feasible solution is that a single element transducer is controlled to rotate around an object. The other one is that linear arrays or ring-shaped arrays are employed for one-time scanning. In the end, PACT images are obtained using state-of-the-art reconstruction algorithms based on the collected PA signals, revealing both the anatomical and functional information of the tissue ${ }^{[13,14]}$.

Some research groups have developed a variety of PACT systems through using different types of the ultrasonic transducers. For example, Zemp et al have developed a piezoelectric transducer array system with a high frequency that includes 48 elements working at a center frequency of $30 \mathrm{MHz}^{[6,15]}$. Similarly, Tan et al have adopted a 48-element transducer to achieve three-dimensional PACT imaging ${ }^{[16]}$. In addition, Tan et al have developed a hemispherical array transducer 
consisting of 256 elements, with a center frequency of $10 \mathrm{MHz}^{[16]}$. Moreover, Zemp et al have reported a fullring array with a diameter of $5 \mathrm{~cm}$ and 512 elements with a center frequency of $5 \mathrm{MHz}^{[15]}$. Furthermore, Nasiriavanaki et al have proposed a PACT imaging system based on a 512-element full-ring ultrasonic transducer array for mouse brain imaging and achieved an in-plane resolution of $100 \mu \mathrm{m}^{[17]}$.

Of note, since the transducers with different center frequencies are generally applied in different biological tissues $^{[18]}$, it reflects the significant importance of the center frequency of the transducer for the PACT imaging quality. In order to enhance the sensitivity of the transducer to the received signal, the PA signals emitted from the tissue need to be matched by the transducer ${ }^{[19]}$. In general, the higher the transducer center frequency is, the more detailed information the image reveals, subsequently providing a higher spatial resolution. In contrast, if low-frequency signals are missing, the brightness and contrast of the image are significantly reduced ${ }^{[20]}$. Therefore, if a low-frequency transducer is used where the high-frequency PA signals cannot be acquired, the produced images will miss detailed information, thus reducing their resolution. To this end, the issue can be addressed by broadening the bandwidth of the ultrasonic transducer to improve the capability of the transducer to collect PA signals with multiple frequencies; however, the manufacturing technique restricts unlimited extension of the transducer bandwidth. Hence, a more practical solution is to incorporate multiple transducers with different center frequencies so that a tradeoff is achieved between image details and brightness/contrast. In this paper, we propose a compact PACT system integrating dual transducers with central frequencies of $2.25 \mathrm{MHz}$ and 7.5 $\mathrm{MHz}$ for image enhancement.

\section{MATERIALS AND METHODS}

\subsection{System Overview}

We designed a compact PACT imaging system equipped with dual ultrasonic transducers, as shown in fig. 1 . The overall system has dimensions of $30 \mathrm{~cm} \times 40$ $\mathrm{cm} \times 60 \mathrm{~cm}$ and consists of three mechanical structures and the corresponding control units.

\subsubsection{Transducer Clamping Devices Distributed on} Both Sides The two single element transducers are clamped into the clamping hole and symmetrically distributed on both ends of the tissue sample. Different detection spacing is chosen based on the samples with different dimensions. The diameter of the detection area is 35-90 $\mathrm{mm}$. For the samples in this size range, the size controller can be manually adjusted, and thereafter imaging steps can be conducted.

1.1.2 Transducer Rotation/Lifting Mechanism The movement of dual transducers mainly involves rotation about the sample. The system setup needs to make the rotation of the transducers occur frequently, and the overall volume of the PACT system is as compact as possible. Therefore, a simple single-axis bearing assembly is used to achieve one-degree-of-freedom rotation of the dual transducers. The whole detection component is implemented based on a bearing with an inner diameter of $120 \mathrm{~mm}$ and an outer diameter of $160 \mathrm{~mm}$. The inner ring of the bearing is fixed, and the transducer holding component and the bearing outer ring are connected to conduct rotational imaging. The entire rotating mechanism is installed on a lifting gear
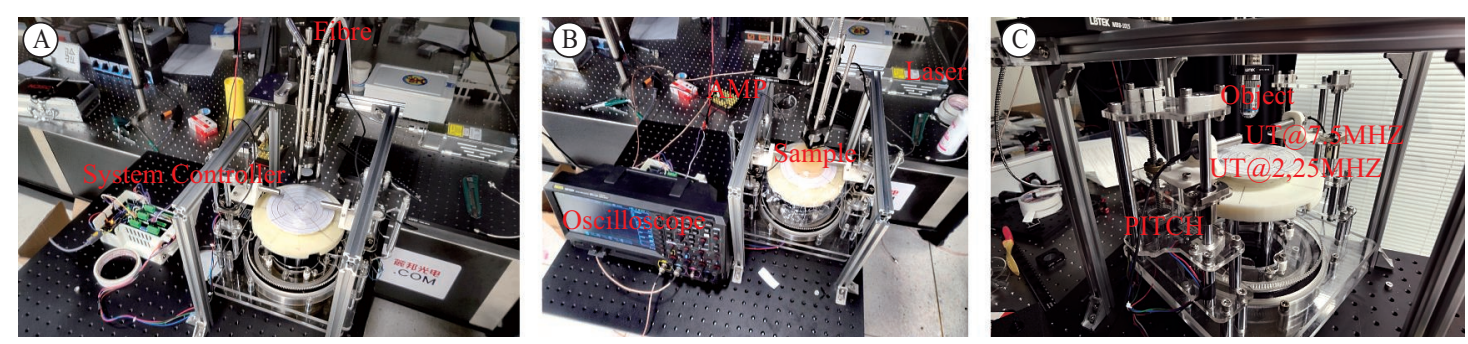

D

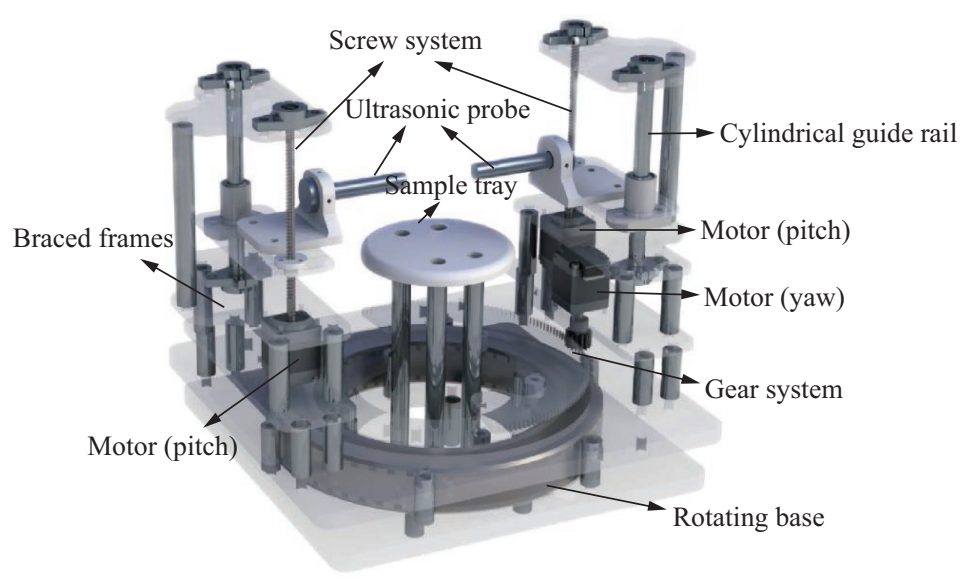

Fig. 1 Photoacoustic computed tomography (PACT) imaging system

A-C: Different views of the system design. D: Three-dimensional diagram of the system. AMP: amplifier; UT: ultrasonic transducer 
to achieve three-dimensional imaging.

\subsubsection{Sample Placement Table Installation of} the sample table and the mounting plate was made co-axially through a smooth shaft and bearing. This installation ensures that sample placement stays steadily such that the samples can be conveniently placed onto the sample erecting bed for imaging.

1.1.4 Platform The platform consists of a bottom rotating part, a lifting part, and a detection frame. The bottom rotating part consists of a 28 stepper motor, a gear meshing structure, a stator frame, and a rotor frame. With the motor and gear combination structure through the stator and rotor, the rotor can be driven to make a circular motion in the plane, which is applied to the probe for arc scanning detection of samples. The lifting part is composed of two 35 stepper motors and a screw rod structure, which is responsible for driving the probe to move in the axial direction of the bottom bearing. The detection frame is responsible for clamping the probe and adjusting the position of the probe.

1.1.5 Arduino Control Platform The system is implemented on an Arduino control platform. The stepper motor is controlled by the Arduino, which sends commands to enable the motor driver to perform a variety of movements. The serial port is used for data communication between the Arduino and the host computer. The computer is used to transmit commands to Arduino via MATLAB (Math Works Inc., USA), and the control platform receives different commands corresponding to different actions of the motor. The movement of the motor drives the probe to reach different positions, where various PA signals are collected.

1.1.6 Overall Pipeline The overall pipeline of the proposed system is illustrated as follows: First, the approximate size of the detected sample is used to estimate the required center frequency of the transducer (e.g., the corresponding frequency of a vessel with a diameter of $0.8 \mathrm{~mm}$ is about $1.25 \mathrm{MHz}$ ). Second, two transducers are symmetrically distributed on both sides, ensuring the same distance between the detection area of each transducer and the center of the sample. In order to generate PA images with high resolution and contrast, a high-frequency transducer and a low-frequency transducer are usually selected simultaneously. A suitable detection radius is selected based on the dimensions of the sample. Furthermore, to confirm a suitable imaging plane for rotational imaging, the bilateral transducers need to be locked. Finally, the number of imaging channels on the host computer is determined, and the system is activated to complete the imaging process.

\subsection{Numerical Simulation}

Tian et al have investigated the influence of the transducer center frequency on PACT imaging performance by conducting both a simulation and experiments. They pointed out that the emitted PA signals from the same tissue have multiple frequency components; nevertheless, the transducer is always concentrated on a certain frequency range in the Fourier frequency domain, which is also known as the transducer center frequency ${ }^{[1,2,19]}$. Thus, the selection of the transducer center frequency is of great importance for improving image quality; however, the experimental results of Tian et al failed to demonstrate the influence of a transducer with multiple frequencies on imaging performance due to the fact that their simulation and experiments are only based on a singlefrequency transducer constraint ${ }^{[19]}$. In this study, we used a K-wave toolkit for PAI simulation to explore the influence of dual transducers with different frequencies on the PACT image quality ${ }^{[2]}$. Initially, two different numerical phantoms were selected (fig. 2A and 2B), where the first one is the numerical phantom of blood vessels with major vessels with a larger diameter than the collateral vessels, and the second one is multiple disks with various diameters (maximum diameter: 2 $\mathrm{mm}$; minimum diameter: $0.2 \mathrm{~mm}$ ). In order to compare the image quality using two dual transducers with that using a single-frequency transducer, we set up three different simulations that contain several combinations of transducer center frequencies, as shown in table 1. In the end, the 256-channel transducer arrays were divided into left and right parts with half channels, respectively.
A

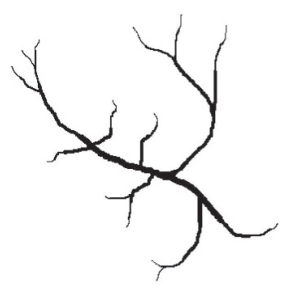

C

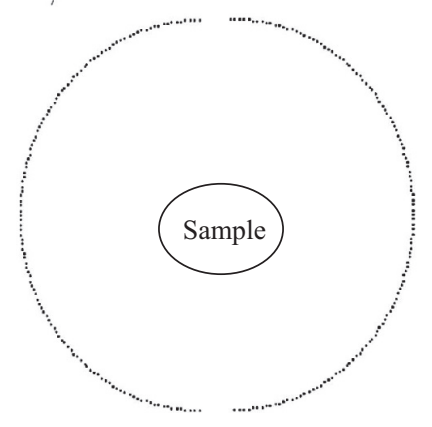

$\mathrm{B}$

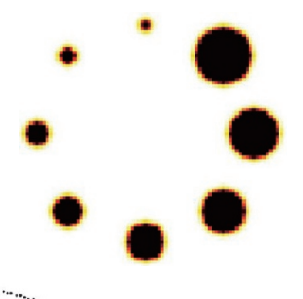

Fig. 2 Schematic diagram of two numerical phantoms

A: vessel phantom; B: multiple disks (maximum diameter:

$2 \mathrm{~mm}$; minimum diameter: $0.2 \mathrm{~mm}$ ); $\mathrm{C}$ : schematic diagram of the transducer array

Table 1 Simulation groups of ultrasonic transducer center frequencies

\begin{tabular}{lcc}
\hline Groups & $\begin{array}{c}\text { Center frequency at left } \\
(\mathrm{MHz})\end{array}$ & $\begin{array}{c}\text { Center frequency at right } \\
(\mathrm{MHz})\end{array}$ \\
\hline $\mathrm{A}$ & 2.25 & 2.25 \\
$\mathrm{~B}$ & 2.25 & 7.5 \\
$\mathrm{C}$ & 7.5 & 7.5 \\
\hline
\end{tabular}




\subsection{Experimental Setup}

To evaluate the efficacy of the overall system, we realized the following system to validate the imaging performance of our proposed PACT system. The schematic diagram of the PACT system is shown in fig. 3. The OPO laser (OPOTEK; repetition frequency, 10 $\mathrm{Hz}$; pulse width, $7 \mathrm{~ns}$; wavelength, $532 \mathrm{~nm}$ ) produces a pulse laser with an energy of about $1 \mathrm{~mJ} /$ pulse source as a signal excitation. First, the light goes into the fiber and passes through the optical fiber collimator (Throlabs, F810APC-543, USA), forming a beam with a diameter of $8 \mathrm{~mm}$. Then, the collimated light enters the scattering plate, whose function is to form a uniform illumination on the top of the sample surface.

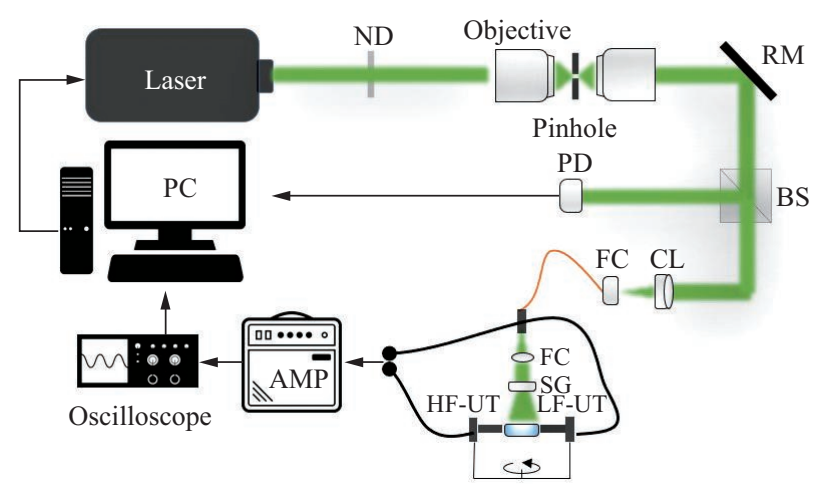

Fig. 3 Schematic of the PAI system setup
After the light is absorbed by an absorber, the absorber emits ultrasonic signals. The ultrasonic signal of each channel is received by a single element transducer with a $60 \%$ bandwidth. The signal-amplifying unit processes the weak signal by providing $36 \mathrm{~dB}$ of gain. Next, the amplified signal is acquired by the data acquisition card (DAQ). In the signal acquisition phase, a bandpass filter with a cut-off frequency from $1 \mathrm{MHz}$ to $10 \mathrm{MHz}$ is employed in order to remove some stray artifacts prior to signal acquisition via the DAQ. The original PA signals emitted from the sample are collected and processed based on a wavelet denoising method to achieve an adaptive threshold selection system ${ }^{[22]}$. Afterwards, a delay and sum algorithm coded in MATLAB (Math Works Inc, USA) is implemented for PA image reconstruction. A host computer is employed to synchronize the overall system, including motor driving, PA signal generation, and data acquisition.

A letter phantom containing " $r$ " and " $\pi$ " was designed to validate our hypothesis. The agar-milk solution with a concentration of about $0.1 \mathrm{~g} / \mathrm{mL}$ was formed, with a scattering coefficient of $48.84 \mathrm{~cm}^{-1}$. Meanwhile, a three-dimensionally printed letter body made of acrylonitrile butadiene styrene was immersed into $1 \%$ black ink (absorption coefficient: $80 \mathrm{~cm}^{-1}$ ) for $2 \mathrm{~h}$ to obtain the abs-ink molding. The solution was poured into the prepared molding, and the three-layer
A

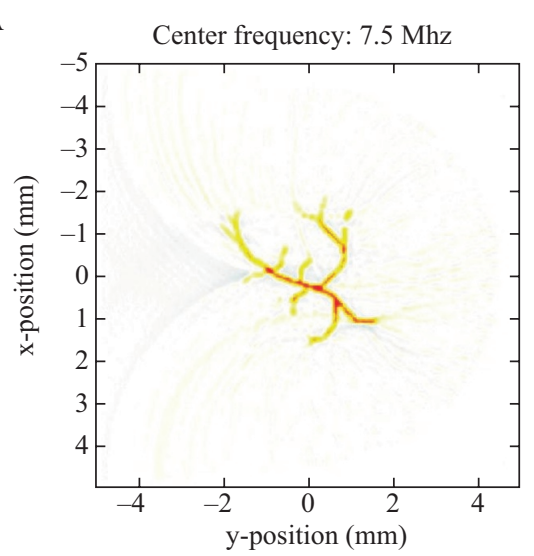

D

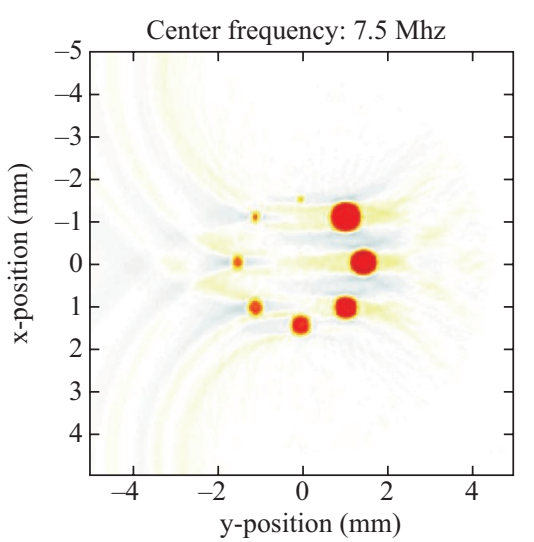

B

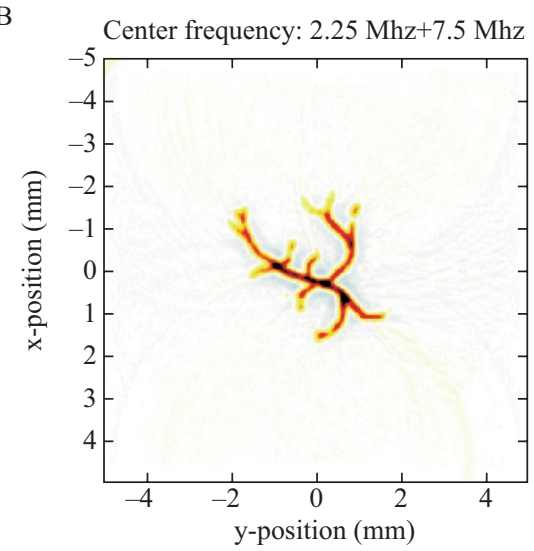

E

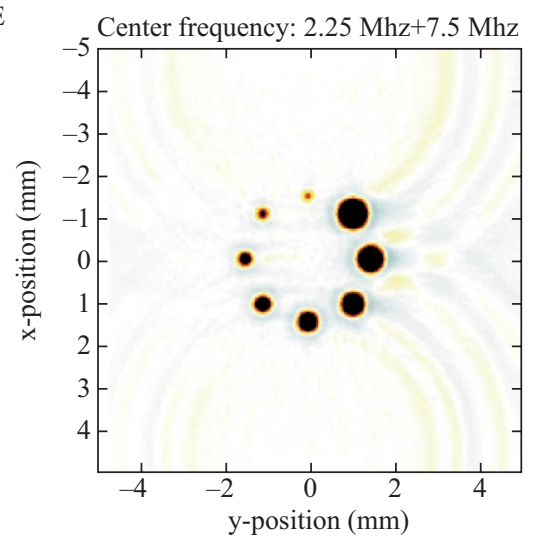

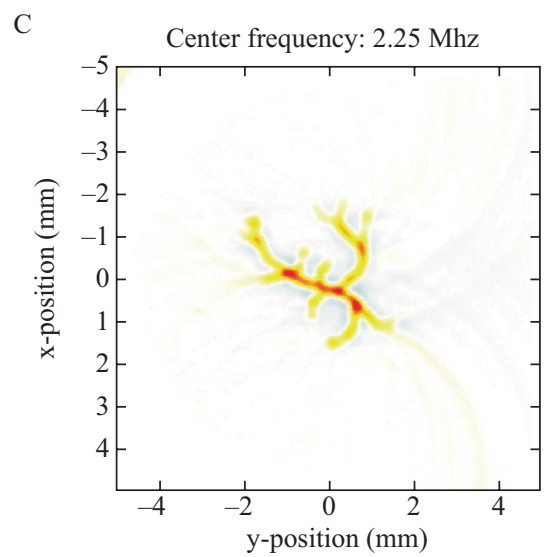

F

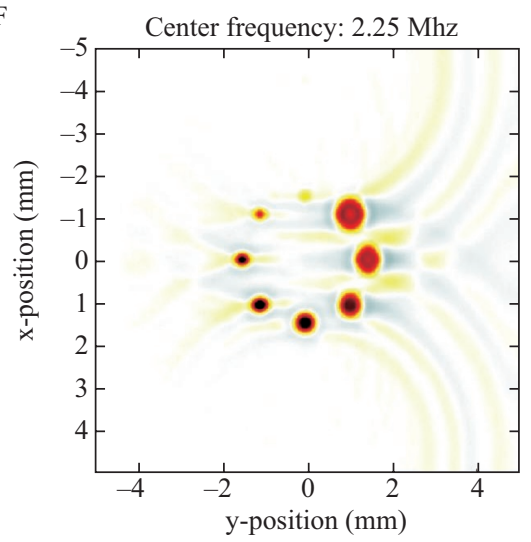

Fig. 4 Simulation results

A, D: simulation results with a center frequency of $7.5 \mathrm{MHz}$ (single); $\mathrm{B}, \mathrm{E}$ : simulation results with center frequencies of $7.5 \mathrm{MHz}$ (half) and $2.25 \mathrm{MHz}$;, $\mathrm{F}$ : simulation results with a center frequency of $2.25 \mathrm{MHz}$ (single) 
structure of the phantom was completely manufactured (fig. 5A and 5E).

\section{RESULTS}

\subsection{Numerical Simulation}

Of note, the transducer center frequency used in PACT needs to match the spatial resolution produced by a tissue sample in order to obtain an optimal signal receiving sensitivity. A smaller absorber results in a higher center frequency. The $2.25-\mathrm{MHz}$ and $7.5-$ $\mathrm{MHz}$ analog transducers were used to conduct fullarea simulation of the numerical phantom (bandwidth of $100 \%$ ). We found that the transducer at $2.25 \mathrm{MHz}$ generated a brighter image with a worse resolution, while the one at $7.5 \mathrm{MHz}$ yielded a better resolution. The reason for this difference is that the loss of lowfrequency components made the PA image darker using the transducer with a higher frequency, which is consistent with our hypothesis.

We used dual transducers with different frequencies to simulate the signal receiving and image reconstruction process. Initially, the condition, the number of transducers is the same as the previous transducer's simulation in a single transducer should be guaranteed, and the only difference should be the transducers' combination with various frequencies. As expected, the combination of dual transducers produced a balance between the brightness and resolution of the PACT, meaning that the PA images produced have a higher resolution in a brighter environment.
However, it should be noted that the dual transducers introduce more background artifacts compared with the experimental results with a detection angle of $360^{\circ}$.

\subsection{Phantom Experiments}

The phantom experiments revealed the PA images of the phantom in different conditions. As shown in fig. $5 \mathrm{~A}$ and $5 \mathrm{D}$, the obtained PA images using a transducer with a center frequency of $7.5 \mathrm{MHz}$ alone have a higher spatial resolution and less artifacts, but they tend to be darker due to the loss of low-frequency information. Similarly, the obtained PA images using a transducer with a center frequency of $2.5 \mathrm{MHz}$ alone have a relatively lower resolution; however, the images are brighter as well (fig. 5C and 5F). These disadvantages can be addressed by incorporating dual transducers covering different center frequencies, thus yielding PA images with both a higher resolution and an ideal image brightness (fig. 5B and 5E).

\section{DISCUSSION}

The influence of the transducer center frequency on imaging performance of the PACT has been reported in previous studies. Our central hypothesis was that dual transducers with different center frequencies can contribute more comprehensive information in PACT imaging due to the fact that dual transducers can create a broader signal frequency-responding range in comparison with a transducer with a single frequency. In this study, we conducted a numerical simulation as well as phantom experiments in order to investigate the
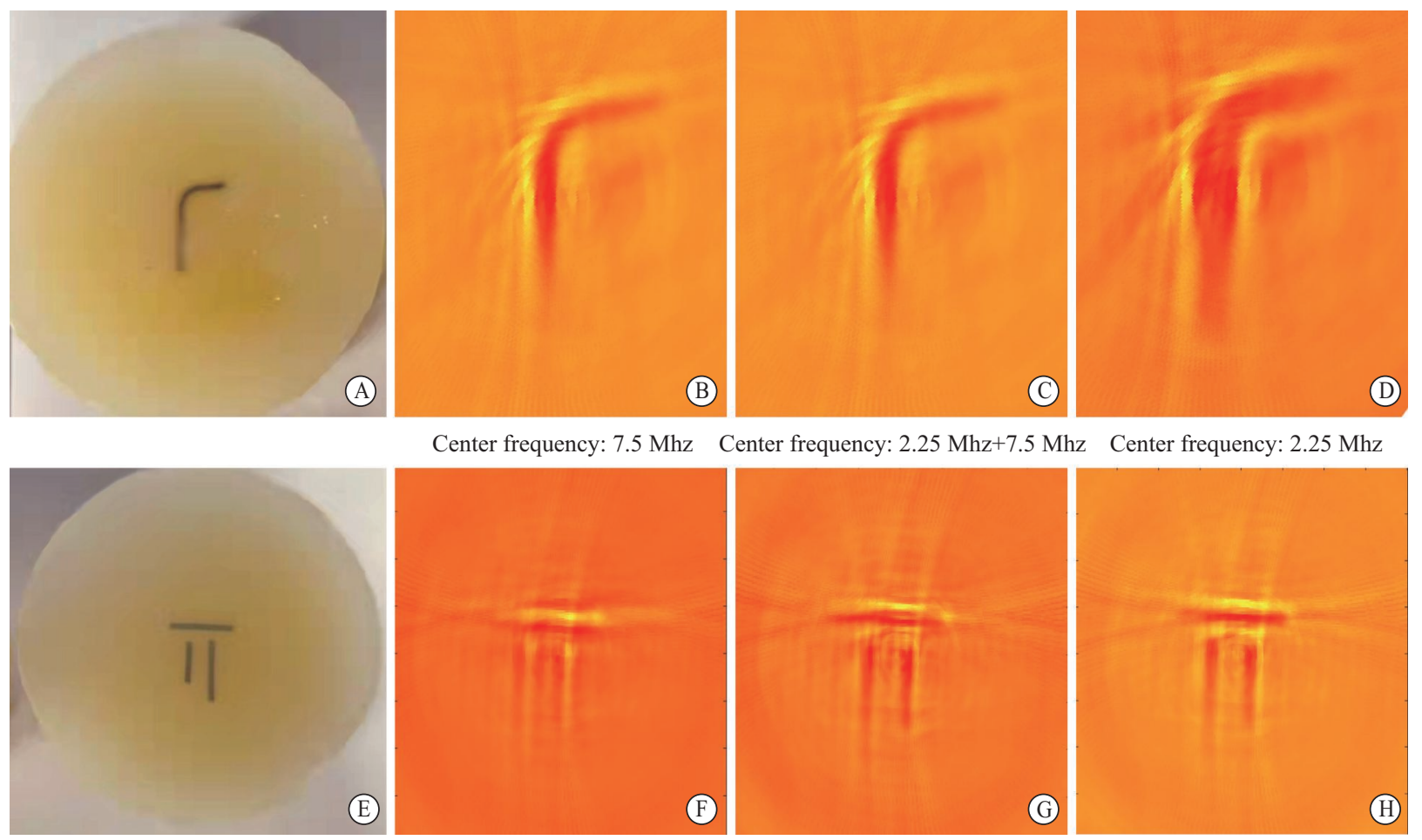

Center frequency: $7.5 \mathrm{Mhz}$

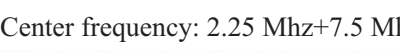

(E)
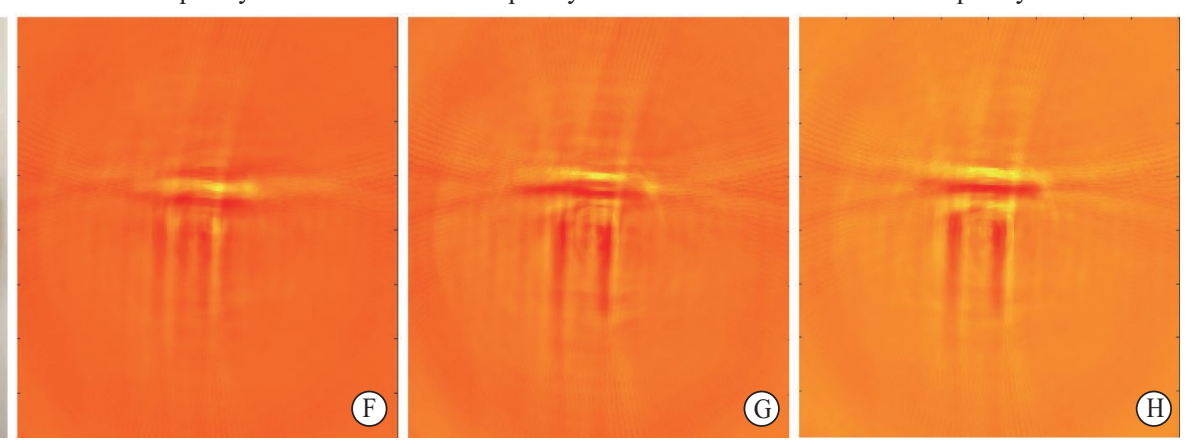

Fig. 5 Photoacoustic imaging (PAI) results

A, E: Phantom design; B, F: the PAI results with a center frequency of $7.5 \mathrm{MHz}$ (single); C, G: the PAI results with center frequencies of $7.5 \mathrm{MHz}$ (half) and $2.25 \mathrm{MHz}$; D, H: the PAI results with a center frequency of $2.25 \mathrm{MHz}$ (single) 
impact of dual transducers on the imaging results. The simulation results demonstrated that the dual transducer configuration can acquire more comprehensive PA signals in comparison with only one transducer with a single frequency whose bandwidth is limited. The phantom experiments confirmed the simulation results as well. When the receiving transducer with a frequency of $7.5 \mathrm{MHz}$ was used alone to receive the PA signals, the entire image exhibited weak brightness due to the loss of low-frequency components of the PA signals. However, by replacing it with a low-frequency transducer, the spatial resolution of the PA image is obviously not ideal. This is because a transducer with a single frequency may be impossible to have an infinite bandwidth for receiving a broader signal range limited by its manufacturing techniques. Thus, when this sort of a single transducer is employed as a PA signal receiver, it inevitably leads to insufficiency of collected signals, subsequently affecting the image quality. Since the received PA signals carry the information emitted from the sample, under the premise that the excitation source remains the same, the frequency of the signals is determined by the physical characteristics of the sample. Thus, signals emitted from a specific sample include multiple frequencies due to the variety of components in the sample. The experimental results demonstrate the potential of the developed compact PACT system with the capability of multiple frequency adjustment, which can produce comprehensive information for clinical translation of PACT imaging.

In this paper, a new PACT system incorporating dual transducer of different frequencies was proposed. The overall dimensions of the system are compact to facilitate portable usage. Compared with the conventional PACT system with a single-frequency transducer, our developed system has a broader range for signal receiving so that it produces more comprehensive information of PA images. This improvement has an important effect on practical applications. In the future, our focus will be on the in vivo image navigation of biomedical microrobots based on our developed dual transducer-enhanced PACT system.

\footnotetext{
Open Access

This article is licensed under a Creative Commons Attribution 4.0 International License https://creativecommons.org/licenses/by/4.0/), which permits use, sharing, adaptation, distribution and reproduction in any medium or format, as long as you give appropriate credit to the original author(s) and the source, provide a link to the Creative Commons licence, and indicate if changes were made. The images or other third party material in this article are included in the article's Creative Commons licence, unless indicated otherwise in a credit line to the material. If material is not included in the article's Creative Commons licence and your intended use is not permitted by statutory regulation or exceeds the permitted use, you will need to
}

obtain permission directly from the copyright holder. To view a copy of this licence, visit http://creativecommons. org/licenses/by/4.0/.

\section{Conflict of Interest Statement}

The authors declare that they have no conflicts of interest.

\section{REFERENCES}

1 Beard P. Biomedical photoacoustic imaging. Interface Focus, 2011,1(4):602-631

2 Tian C, Zhang C, Zhang H, et al. Spatial Resolution in Photoacoustic Computed Tomography. Rep Prog Phys, 2021,84(3):036701

3 Guo H, Li Y, Qi W, et al. Photoacoustic endoscopy: A progress review. JBiophotonics, 2020,13(12):e202000217

4 Wang LV, Yao J. A practical guide to photoacoustic tomography in the life sciences. Nat Methods, 2016, 13(8):627-638

5 Steinberg I, Huland DM, Vermesh O, et al. Photoacoustic clinical imaging. Photoacoustics, 2019,14:77-98

6 Li C, Wang LV. Photoacoustic tomography and sensing in biomedicine. Phys Med Biol, 2009,54(19):R59-97

7 Wang LV, Wu SI. Biomedical Optics: Principles and Imaging. Hoboken: Wiley Press, 2007:201-236

8 Gao F, Feng X, Zheng Y. Advanced photoacoustic and thermoacoustic sensing and imaging beyond pulsed absorption contrast. J Optics, 2016,18(7):074006

9 Gao F, Feng X, Zheng Y. Coherent photoacousticultrasound correlation and imaging. IEEE Trans Biomed Eng, 2014,61(9):2507-2512

10 Attia ABE, Balasundaram G, Moothanchery M, et al. A review of clinical photoacoustic imaging: Current and future trends. Photoacoustics, 2019,16:100144

11 Fatima A, Kratkiewicz K, Manwar R, et al. Review of cost reduction methods in photoacoustic computed tomography. Photoacoustics, 2019,15:100137

12 Shi J, Tang Y, Yao J. Advances in super-resolution photoacoustic imaging. Quant Imaging Med Surg, 2018, 8(8):724-732

13 Liu S, Feng X, Jin H, et al. Handheld Photoacoustic Imager for Theranostics in 3D. IEEE Trans Med Imaging, 2019,38(9):2037-2046

14 Zhou Y, Yao J, Wang LV. Tutorial on photoacoustic tomography. J Biomed Opt, 2016,21(6):61007

15 Zemp R, Bitton R, Shung K, et al. Photoacoustic imaging of the microvasculature with a high-frequency ultrasound array transducer. J Biomed Optics, 2007, 12(1):010501

16 Tan Y, Xia K, Ren Q, et al. Three-dimensional photoacoustic imaging via scanning a one dimensional linear unfocused ultrasound array. Opt Express, 2017, 25(7):8022-8028

17 Nasiriavanaki M, Xia J, Wan H, et al. High-resolution photoacoustic tomography of resting-state functional connectivity in the mouse brain. Proc Natl Acad Sci USA, 2014,111(1):21-26

18 Wang $\mathrm{X}$, Pang $\mathrm{Y}, \mathrm{Ku} \mathrm{G}$, et al. Noninvasive laserinduced photoacoustic tomography for structural and functional in vivo imaging of the brain. Nat Biotechnol, 2003,21(7):803-806

19 Tian C, Pei M, Shen K, et al. Impact of System Factors 
on the Performance of Photoacoustic Tomography Scanners. Phys Rev Applied, 2020,13(1): 014001

20 Wang LV, Song H. Photoacoustic tomography: in vivo imaging from organelles to organs. Science (New York, N.Y.) , 2018,335(6075):1458-1462

21 Treeby BE, Cox BT: k-Wave: MATLAB toolbox for the simulation and reconstruction of photoacoustic wave fields. J Biomed Opt, 2010,15(2):021314
22 Zhou M, Xia $\mathrm{H}$, Lan $\mathrm{H}$, et al. In Wavelet denoising method with adaptive threshold selection for photoacoustic tomography, 2018 40th Annual International Conference of the IEEE Engineering in Medicine and Biology Society (EMBC), 18-21 July 2018: 4796-4799

(Received Oct. 27, 2021; accepted Nov. 19, 2021) 